\title{
The Probabilistic Instantaneous Matching Algorithm
}

\author{
Frederik Beutler and Uwe D. Hanebeck
}

\begin{abstract}
A new Bayesian filtering technique for estimating signal parameters directly from discrete-time sequences is introduced. The so called probabilistic instantaneous matching algorithm recursively updates the probability density function of the parameters for every received sample and, thus, provides a high update rate up to the sampling rate with high accuracy. In order to do so, one of the signal sequences is used as part of a time-variant nonlinear measurement equation. Furthermore, the time-variant nature of the parameters is explicitly considered via a system equation, which describes the evolution of the parameters over time. An important feature of the probabilistic instantaneous matching algorithm is that it provides a probability density function over the parameter space instead of a single point estimate. This probability density function can be used in further processing steps, e.g. a range based localization algorithm in the case of time-of-arrival estimation.
\end{abstract}

\section{INTRODUCTION}

This article presents a new probabilistic approach for estimating unknown time-variant signal parameters from a set of discrete-time sequences. The proposed approach will be exemplified for the special case of time delay estimation, which arises in applications like target tracking or localization. In that case, the desired parameter is the time delay estimated based on the signal emitted by a source and received by a sink, which is subsequently used by e.g. range based localization approaches [1], [2].

Standard approaches for time delay estimation like blockwise algorithms, e.g. the well known cross-correlation [3], or recursive algorithms, e.g. adaptive filters, neglect uncertainties inherent in the signals and do not explicitly consider the time-variant nature of the signal parameters. An adaptive filter is a recursive algorithm, that adjusts the filter coefficients in such a way that the difference between the measured and the filtered samples is minimized. This allows consideration of slowly time-varying parameters. However, the adaptation parameter has to be set in such a way that the algorithm does not become unstable. For cross-correlation, where information processing is performed in a block-wise manner, the block length should maximize the signal-tonoise ratio and minimize the peak smearing, which occurs if the sink or the source is moving. Several more advanced approaches based on cross-correlation have been introduced. An example is region based cross-correlation [4], where the template is split into different regions. In [5] an extended matched filter is used for time delay estimation. To handle overlapping echoes, a parallel bank of different matched

F. Beutler and U. D. Hanebeck are with the Intelligent Sensor-ActuatorSystems Laboratory, Institute of Computer Science and Engineering, Universität Karlsruhe (TH), Germany.

\{Beutler|Uwe. Hanebeck\}@ieee. org filters are used, where the signal is modeled as a nonstationary auto covariance function. A maximum-likelihood estimator for time delay estimation is presented in [6].

Some modern approaches describe cross-correlation in a probabilistic way. In [7] and [8] the so called Bayesian correlation algorithm for object localization in images is introduced. This Bayesian approach works in the feature space and not in the signal domain as our approach. In [9] a method for time delay estimation using Bayesian regularization and nonnegative deconvolution is presented.

This paper introduces a Bayesian filtering technique for estimating parameter, e.g. the time delay, directly from time sequences that explicitly considers uncertainties and provides a probability density function over the desired parameter space. In addition, a model for the evolution of the desired parameter over time and its associated uncertainty can be used. A major novelty is the interpretation of a known reference sequence as part of a nonlinear measurement equation of a nonlinear system.

The new so called probabilistic instantaneous matching algorithm immediately processes every given signal sample in a recursive fashion. In contrast, conventional methods, e.g. cross-correlation, estimate the time delay from overlapping or consecutive blocks of samples. Hence, the computational complexity of the proposed new approach is much lower. In addition, processing delay is significantly reduced.

The implementation of the probabilistic instantaneous matching algorithm is based on an efficient representation of the probability density functions by means of Gaussian mixtures. In order to use Gaussian mixtures for representing the likelihood function, one signal is part of a time-variant measurement equation, where the values are linearly interpolated between two samples. In order to limit the number of mixture components, the prediction step is performed based on an approximation of the underlying transition density by means of an axis-aligned Gaussian mixture.

The structure of this paper is as follows. A problem formulation for estimating the parameter between two time signals is presented in Sec. II. Furthermore, the generic Bayesian estimator is introduced. In Sec. III the measurement model is derived. This measurement model is used in Sec. IV to determine the probabilistic model. The filter algorithm is divided into two parts. In Sec. V the measurement update and in Sec. VI the time update is explained. The performance of the new approach compared to the traditional solution is evaluated in Sec. VII. Simulation and experimental results are also shown. Conclusions and details on future investigations are given in Sec. VIII. 


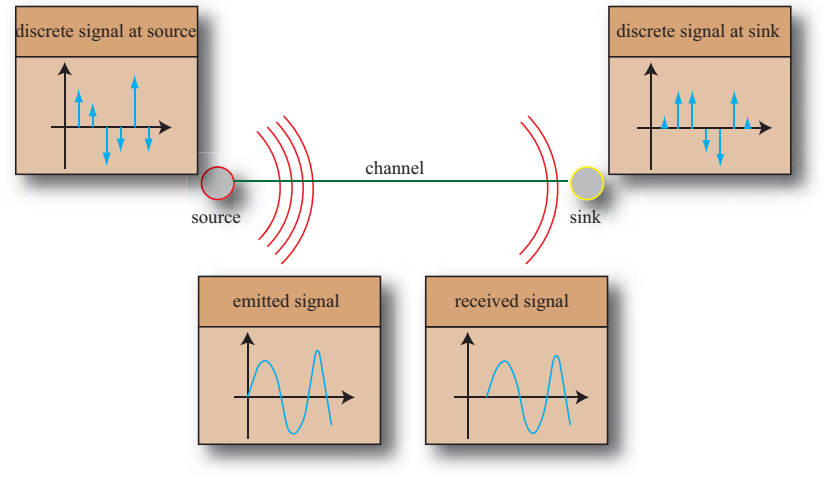

Fig. 1. A source/sink-model.

\section{Problem Formulation}

A discrete-time signal $s_{d}(t)$ can be described as a weighted Dirac mixture

$$
s_{d}(t)=\sum_{k} c_{k} \delta(t-k T),
$$

where $T$ is the sampling interval. If the signal is emitted by a source, the output signal $s(t)$ is a continuous-time signal. The output signal is filtered with a partially unknown time-variant system $h(\tau, t)$. In addition, the filtered signal is corrupted with additive noise $v(t)$. A sink receives this signal $y(t)$, which can be described as

$$
y(t)=\int_{-\infty}^{+\infty} s(t-\tau) h(\tau, t) \mathrm{d} \tau+v(t) .
$$

The received signal $y(t)$ is sampled, so that at each discrete time step $l T$ a single amplitude measurement $y(l T)$ is given. From these sequentially received amplitude measurements the parameters of the time-variant system $h(\tau, t)$ have to be estimated. These parameters include for example the time delay, which results from the distance between a microphone and a loudspeaker. In Fig. 1 a source/sink-model is illustrated.

In order to estimate the time delay between two time signals $s(t)$ and $y(t)$, a Bayesian filtering technique is used. In general the Bayesian filtering algorithm consists of a measurement and a system update. The measurement equation as well as the system equation have to be identified. For an amplitude measurement $y_{l}$ at time $l T$ the measurement update is given by

$$
f_{l}^{e}\left(\tau_{l}\right)=c f_{l}^{L}\left(y_{l} \mid \tau_{l}\right) f_{l}^{p}\left(\tau_{l}\right)
$$

where $f_{l}^{e}\left(\tau_{l}\right)$ is the estimated density function, $f_{l}^{L}\left(y_{l} \mid \tau_{l}\right)$ the likelihood function and $c$ a normalization constant. The predicted density function $f_{l}^{p}\left(\tau_{l}\right)$ represents prior knowledge about the estimated time delay. In the time update a new predicted density is calculated according to

$$
f_{l}^{p}\left(\tau_{l+1}\right)=\int_{\mathbb{R}} f^{T}\left(\tau_{l+1} \mid \tau_{l}\right) \cdot f_{l}^{e}\left(\tau_{l}\right) d \tau_{l},
$$

where $f_{l}^{e}\left(\tau_{l}\right)$ is the estimated density from the measurement update and $f^{T}\left(\tau_{l+1} \mid \tau_{l}\right)$ is the transition density, which depends on the system equation. The system equation that describes the propagation of the parameters to the next time step can be written as

$$
\tau_{l+1}=a\left(\tau_{l}\right)+w_{l},
$$

where $a($.$) is the nonlinear system equation and w_{l}$ is the additive process noise.

\section{IDENTIFICATION OF THE MEASUREMENT EQUATION}

The discrete-time signal given in (1), where $c_{k}$ is the amplitude value at time step $k$ and $T$ is the sampling interval, can be interpolated to get a continuous-time signal. The sample values $c_{k}$ and $c_{k+1}$ are linearly interpolated and so the signal $s(t)$ is given by

$$
\begin{aligned}
& s(t) \\
& =\sum_{k}\left(\frac{c_{k+1}-c_{k}}{T} t+(1+k) c_{k}-k c_{k+1}\right) \operatorname{rect}\left(\frac{t}{T}-\frac{1}{2}-k\right),
\end{aligned}
$$

where

$$
\operatorname{rect}(t)= \begin{cases}1 & |t|<\frac{1}{2} \\ 0 & \text { otherwise }\end{cases}
$$

is the rectangular function. By substituting

$$
a_{k}=\frac{c_{k+1}-c_{k}}{T}
$$

and

$$
b_{k}=(1+k) c_{k}-k c_{k+1},
$$

the signal can be simplified to

$$
s(t)=\sum_{k}\left(a_{k} t+b_{k}\right) \operatorname{rect}\left(\frac{t}{T}-\frac{1}{2}-k\right) .
$$

The time-variant system $h(\tau, t)$, which describes the relationship between the input signal $s(t)$ and the output signal $y(t)$ can be described as in (2). The function $h(\tau, t)$ includes the time-variant sink position $p(t)$, the time-variant source position $\underline{x}(t)$ and the velocity of propagation $c$. Under the assumption of no reflections, a simple model for $h(\tau, t)$ is

$$
h(\tau, t)=d(\underline{p}(t), \underline{x}(t), c) \delta(\tau-f(\underline{p}(t), \underline{x}(t), c)),
$$

where $f($.$) is a nonlinear function which describes the time$ delay depending on the distance between sink and source position. The function $d($.$) is an attenuation term, which is$ assumed to be constant. The simple model from (4) is used in (2) resulting in

$$
y(t)=\int_{-\infty}^{+\infty} s(t-\tau) \delta(\tau-f(\underline{p}(t), \underline{x}(t), c)) \mathrm{d} \tau+v(t) .
$$

With the properties of the Dirac delta function it can be simplified to

$$
y(t)=s(t-f(\underline{p}(t), \underline{x}(t), c))+v(t) .
$$

The nonlinear function $f($.$) is substituted by the time delay$ $\tau(t)$

$$
\left.f\left(\underline{p}_{i}(t), \underline{x}(t), c\right)\right)=\tau(t)
$$


and a measurement equation

$y(t)=\sum_{k}\left(a_{k}(t-\tau(t))+b_{k}\right) \operatorname{rect}\left(\frac{t-\tau(t)}{T}-\frac{1}{2}-k\right)+v(t)$

for a certain sink given. For a causal system, a single measurement at time $l T$ is given as

$$
\begin{aligned}
& y(l T) \\
& =\sum_{\substack{k=0 \\
+}}^{l}\left(a_{k}(l T-\tau(l T))+b_{k}\right) \operatorname{rect}\left(\frac{l T-\tau(l T)}{T}-\frac{1}{2}-k\right) \\
& +v(l T) .
\end{aligned}
$$

If we assume, that the time delay is limited, only an interval for $\tau \in\left[0, \tau_{\max }\right]$ can be considered. The value for $\tau_{\max }$ is converted to a discrete-time value $D=\operatorname{round}\left(\frac{\tau_{\max }}{T}\right)$. The resulting measurement equation for a single amplitude measurement is

$$
\begin{aligned}
& y(l T) \\
& =\sum_{\substack{k=l-D \\
+v(l T)}}^{l}\left(a_{k}(l T-\tau(l T))+b_{k}\right) \operatorname{rect}\left(\frac{l T-\tau(l T)}{T}-\frac{1}{2}-k\right) \\
&
\end{aligned}
$$

For simplicity, the time index $l T$ is put into subscript as $(.)_{l}$, so the measurement equation results in

$y_{l}=\sum_{k=l-D}^{l}\left(a_{k}\left(l T-\tau_{l}\right)+b_{k}\right) \operatorname{rect}\left(\frac{l T-\tau_{l}}{T}-\frac{1}{2}-k\right)+v_{l}$.

\section{Derivation of a Probabilistic Model}

According to the measurement equation in (5), a generative model suffering from additive noise, a likelihood function $f^{L}$ is given by

$$
\begin{aligned}
& f_{l}^{L}\left(y_{l} \mid \tau_{l}\right)= \\
& f^{v}\left(y_{l}-\sum_{k=l-D}^{l}\left(a_{k}\left(l T-\tau_{l}\right)+b_{k}\right) \operatorname{rect}\left(\frac{l T-\tau_{l}}{T}-\frac{1}{2}-k\right)\right) .
\end{aligned}
$$

We assume the density $f^{v}$ to be Gaussian, resulting in

$$
\begin{aligned}
& f_{l}^{L}\left(y_{l} \mid \tau_{l}\right)= \\
& \mathrm{N}\left(y_{l}-\sum_{k=l-D}^{l}\left(a_{k}\left(l T-\tau_{l}\right)+b_{k}\right) \operatorname{rect}\left(\frac{l T-\tau_{l}}{T}-\frac{1}{2}-k\right), \sigma_{v}\right) .
\end{aligned}
$$

This function is piecewise Gaussian, as the rectangular functions do not overlap. Hence, the sum and the rectangular functions can be pulled out, so that the likelihood function can be simplified to

$$
\begin{aligned}
& f_{l}^{L}\left(y_{l} \mid \tau_{l}\right)= \\
& \sum_{k=l-D}^{l} \frac{1}{\sqrt{2 \pi} \sigma_{v}} \exp \left(-0.5\left(\frac{\tau_{l}-\mu_{k}}{\sigma_{k}}\right)^{2}\right) \operatorname{rect}\left(\frac{\tau_{l}}{T}+0.5+k-l\right)
\end{aligned}
$$

with

$$
\mu_{k}=\frac{-y_{l}+a_{k} l T+b_{k}}{a_{k}}
$$

and

$$
\sigma_{k}=\frac{\sigma_{v}}{a_{k}}
$$

For efficient implementation, the rectangular function can be approximated with a Gaussian mixture according to

$$
\operatorname{rect}\left(\frac{\tau_{l}}{T}+0.5+k-l\right)=\sum_{j=1}^{M} w_{j} \mathrm{~N}\left(\tau_{l}-\mu_{k}^{j}, \sigma^{j}\right),
$$

with the weighting factors

$$
w_{j}=\frac{T}{M},
$$

expected values

$$
\mu_{k}^{j}=(l-k-1) T+(j-0.5) \frac{T}{M}
$$

and standard derivations

$$
\sigma^{j}=\sigma \frac{T}{M} .
$$

This approximation of the rectangular function is substituted in (6). The resulting expression is a multiplication of Gaussian mixtures

$$
\begin{aligned}
& f_{l}^{L}\left(y_{l} \mid \tau_{l}\right) \\
& =\sum_{k=l-D}^{l} \frac{1}{\sqrt{2 \pi} \sigma_{v}} \exp \left(-0.5\left(\frac{\tau_{l}-\mu_{k}}{\sigma_{k}}\right)^{2}\right) \sum_{j=1}^{M} w_{j} \mathrm{~N}\left(\tau_{l}-\mu_{k}^{j}, \sigma^{j}\right) \\
& =\sum_{k=l-D}^{l} \sum_{j=1}^{M} \underbrace{w_{k}}_{\frac{\sigma_{k}}{\sigma_{v}}} \mathrm{~N}\left(\tau_{l}-\mu_{k}, \sigma_{k}\right) w_{j} \mathrm{~N}\left(\tau_{l}-\mu_{k}^{j}, \sigma^{j}\right) .
\end{aligned}
$$

The multiplication result can be combined to a Gaussian mixture with $M \cdot(D+1)$ components. The resulting likelihood function is given by

$$
f_{l}^{L}\left(y_{l} \mid \tau_{l}\right)=\sum_{u=0}^{M \cdot D} w_{u}^{g} \mathrm{~N}\left(\tau_{l}-\mu_{u}^{g}, \sigma_{u}^{g}\right),
$$

where the components are calculated by the following expressions

$$
\begin{gathered}
\mu_{u}^{g}=\frac{\mu_{k} \sigma^{j^{2}}+\mu_{k}^{j} \sigma_{k}^{2}}{\sigma^{j^{2}+\sigma_{k}^{2}}}, \\
\sigma_{u}^{g}=\sqrt{\frac{\sigma^{j^{2} \sigma_{k}^{2}}}{\sigma^{j^{2}}+\sigma_{k}^{2}}}
\end{gathered}
$$

and

$$
w_{u}^{g}=\frac{w_{k} w_{j}}{\sqrt{2 \pi\left(\sigma^{j^{2}}+\sigma_{k}^{2}\right)}} \exp \left(-\frac{1}{2} \frac{\left(\mu_{k}-\mu_{k}^{j}\right)^{2}}{\sigma^{j^{2}}+\sigma_{k}^{2}}\right),
$$

with $k=l-D, \ldots, l$ and $j=1, \ldots, M$.

\section{Measurement Update}

In the measurement update, the estimated density $f_{l}^{e}\left(\tau_{l}\right)$ will be calculated according to Bayes' law

$$
f_{l}^{e}\left(\tau_{l}\right)=c f_{l}^{L}\left(y_{l} \mid \tau_{l}\right) f_{l}^{p}\left(\tau_{l}\right) .
$$

As stated above, the likelihood function consists of $M \cdot(D+$ 1) Gaussian mixture components and the predicted density $f_{l}^{p}\left(\tau_{l}\right)$ is a Gaussian mixture with $N$ components, so the estimated density $f_{l}^{e}\left(\tau_{l}\right)$ has $N \cdot(M \cdot(D+1))$ Gaussian mixture components. For calculating the components of the estimated density $\mu_{i}, \sigma_{i}, w_{i}$ with $i=1, \ldots, N \cdot(M \cdot(D+1))$ (7), (8) and (9) can be used. To obtain a valid density the estimated density has to be normalized. 


\section{TIME UPDATE}

In analogy to the measurement equation, a system equation, which is given in (3), has to be identified. In this paper we consider a simple linear model with additive time invariant process noise $w_{l}$ according to

$$
\tau_{l+1}=\tau_{l}+w_{l} .
$$

Thus the uncertainty of the parameter is increased over time. According to the given system equation the transition density is given by

$$
f^{T}\left(\tau_{l+1} \mid \tau_{l}\right)=\int_{\mathbb{R}} \delta\left(\tau_{l+1}-\tau_{l}\right) f^{w}(w) \mathrm{d} w=f^{w}\left(\tau_{l+1}-\tau_{l}\right) .
$$

The process noise $f^{w}$ is assumed to be Gaussian and the transition density is approximated with an axis-aligned Gaussian mixture with $N$ components

$$
f^{T}\left(\tau_{l+1} \mid \tau_{l}\right)=\sum_{i=1}^{N} w_{i} N\left(\tau_{l+1}-\mu_{i}, \sigma_{i}\right) N\left(\tau_{l}-\mu_{i}, \sigma_{i}\right) .
$$

In order to use a more realistic process noise model, the density can be approximated with a Gaussian mixture. In this case the number of the components of the resulting transition density is increasing depending on the approximation of the process noise. If a nonlinear transition density model is used, it can be approximated with an algorithm, which is described in [10]. By using this transition density the predicted density

$f_{l}^{p}\left(\tau_{l+1}\right)=\int_{\mathbb{R}} \sum_{i=1}^{N} w_{i} N\left(\tau_{l+1}-\mu_{i}, \sigma_{i}\right) N\left(\tau_{l}-\mu_{i}, \sigma_{i}\right) f_{l}^{e}\left(\tau_{l}\right) \mathrm{d} \tau_{l}$ can be written as

$$
\begin{aligned}
& f_{l}^{p}\left(\tau_{l+1}\right) \\
& =\sum_{i=1}^{N} N\left(\tau_{l+1}-\mu_{i}, \sigma_{i}\right) \underbrace{w_{i} \int_{\mathbb{R}} N\left(\tau_{l}-\mu_{i}, \sigma_{i}\right) f_{l}^{e}\left(\tau_{l}\right) \mathrm{d} \tau_{l}}_{w_{i}^{p}} .
\end{aligned}
$$

That means, that only the weighting factors of the components for $\tau_{l+1}$ have to be updated. The resulting predicted density has $N$ components.

\section{RESULTS}

The performance of the new approach is evaluated in simulation as well as in experiments with real data. Furthermore, the new algorithm will be compared with the conventional method, the classic cross-correlation.

\section{A. Simulation Results}

The performance of the new approach is evaluated by simulations in a two-dimensional coordinate system. In the simulation setup a moving source is modeled. The motion of the source is implemented with a piecewise constant velocity. The source signal is delayed depending on the time-variant distance between sink and source. The signals are generated with zero-mean white Gaussian noise with unit variance. In addition, the signals are disturbed by adding zero-mean white Gaussian noise with different variances. Equation (2) is used to generate the received samples, where the timevariant system is given by

$$
h(\tau, t)=\operatorname{sinc}(\tau-f(\underline{p}(t), \underline{x}(t), c))
$$

and the nonlinear function $f($.$) is given by$

$$
f(\underline{p}(t), \underline{x}(t), c)=\frac{|\underline{p}(t)-\underline{x}(t)|_{2}}{c} .
$$

The sampling frequency is set to $f_{t}=4800 \mathrm{~Hz}$. For the new approach, the parameters for the likelihood function and for the transition density were selected as follows.

For the likelihood function the maximum time delay is $\tau_{\max }=0.0035$ seconds. The measurement noise is set to $\sigma_{v}=1$. Each rectangular function is approximated by $M=10$ components and the variance is set to $\sigma=0.7$. Each variance of the likelihood function depends on the time-variant amplitude values. The variance decreases if the distance between the amplitude values increases. Obviously, the variance depends on the frequency spectrum of the signal. The approximation of the rectangular function is sufficient. However, each approximated rectangular function overlap, which results in an error for the likelihood function.

The transition density is approximated by $N=60$ components. The expected values are linearly equally spaced points between $\mu_{t r}=[0, \ldots, 0.0035]$ seconds. The variance of the transition density is set to $\sigma_{t r}=1.52 \cdot 10^{-5}$. The variance of the transition density has to be adjusted with respect to the sampling frequency, because in the time update only the weighting factors of the predicted density are updated.

For cross-correlation the block length is set to 50 samples. The cross-correlation is calculated for every sample, which means that the block is shifted when a sample is received. Based on the results of cross-correlation the maximum value is then selected.

In the simulations the variance of the noise term is $0,0.5$ and 1 , respectively. The results are shown in Fig. 2(a-b), where the new approach (blue line), cross-correlation (red line) and the true time delay (black line) are illustrated. The new approach has a transition time of 14 samples, where cross-correlation provides a result after 50 samples. Furthermore, cross-correlation produces outliers, when the noise increases. The new approach does not produce outliers, because previous information represented in the predicted density is used for estimation. The squared error between the true time delay and the estimates is illustrated in Fig. 2(d-f). The squared error of the estimates from the new approach is smaller than from cross-correlation. Even if the noise level is very high the squared error of the estimates from the new approach is lower than $10^{-8}$.

\section{B. Experimental Results}

In the real experimental setup, a source moves for three seconds towards the sink and back to the starting point. The maximum distance deviation is measured by hand as 0.34 meters. The velocity of sound is assumed to $343 \mathrm{~m} / \mathrm{s}$, thus the distance deviation is around $\tau_{\text {deviation }}=0.99125 \cdot 10^{-3}$ seconds. In Fig. 3 the results are shown. The sampling 

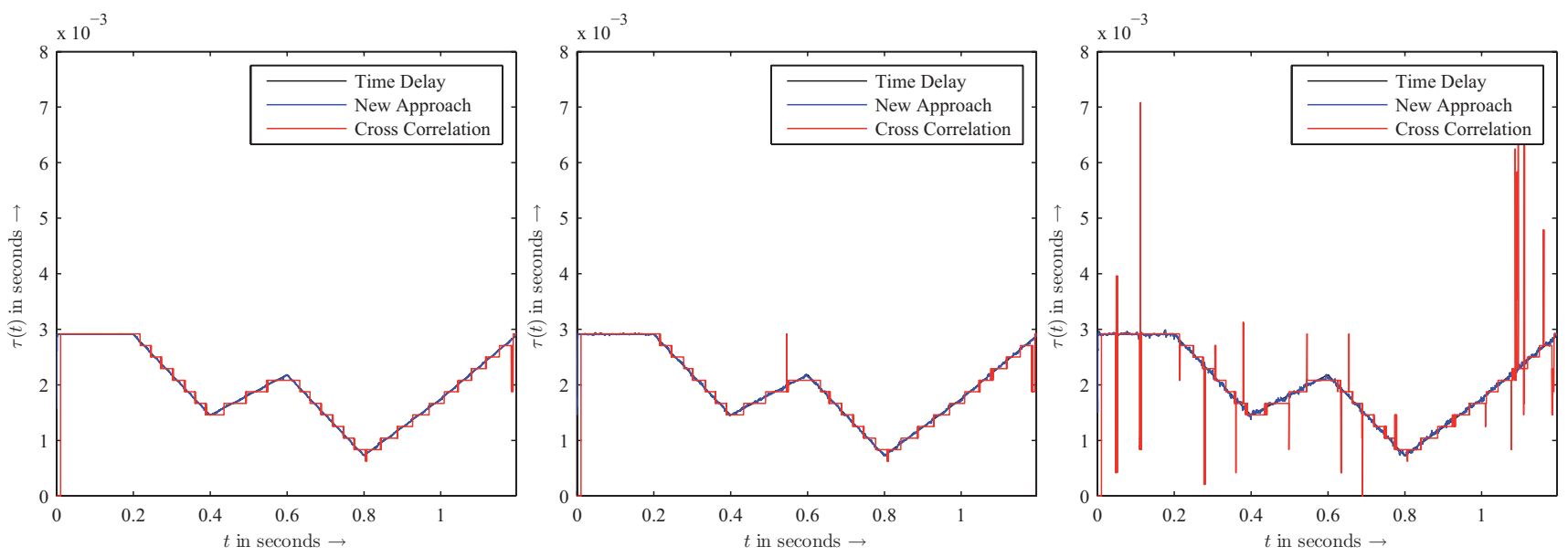

(a) Comparison of the new algorithm and the con- (b) Comparison of the new algorithm and the con- (c) Comparison of the new algorithm and the conventional method in the simulation with a noise ventional method in the simulation with a noise ventional method in the simulation with a noise level $\sigma_{v}=0$.

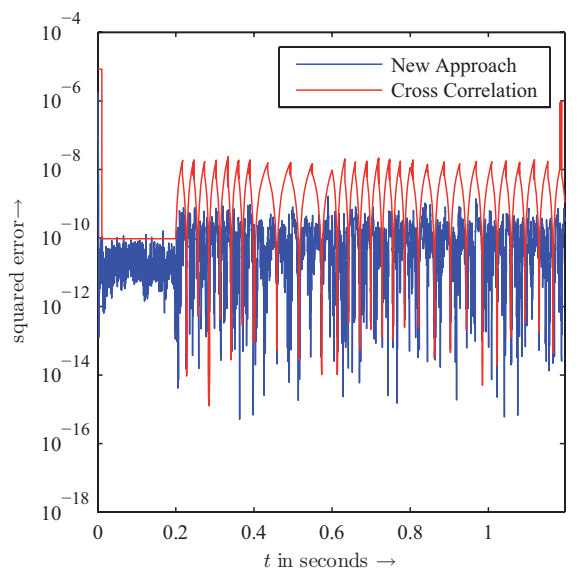

(d) Squared error by noise level $\sigma_{v}=0$.

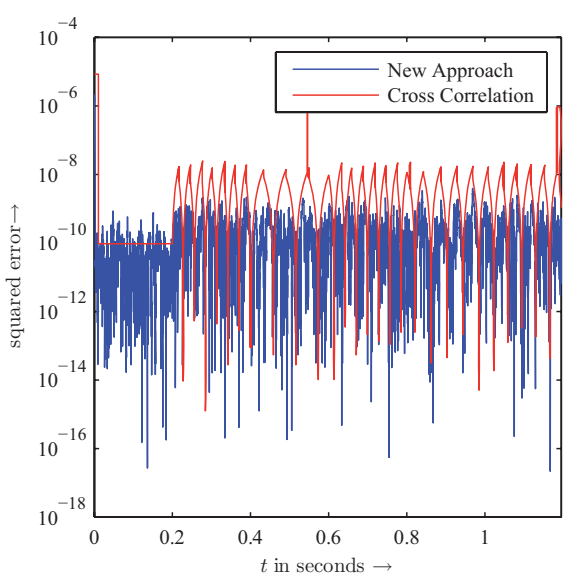

(e) Squared error by noise level $\sigma_{v}=0.5$.

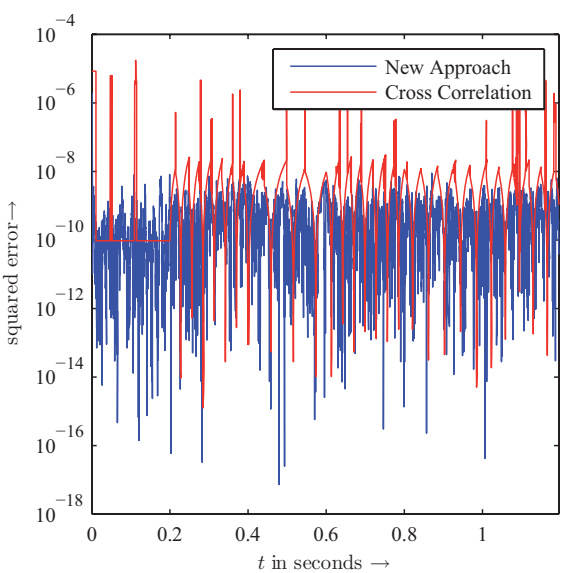

(f) Squared error by noise level $\sigma_{v}=1$.

Fig. 2. Simulation result for a moving source. The classic cross-correlation is compared with new probabilistic approach.

frequency is $48000 \mathrm{~Hz}$. The parameters for the new approach are as follows.

The maximum time delay of the likelihood function is $\tau_{\max }=0.0035$ seconds. The measurement noise is set to $\sigma_{v}=0.5$. Each rectangular function is approximated by $M=10$ components and the variance is set to $\sigma=0.7$.

The transition density is approximated by $N=200$ components. The expected values are linearly equally spaced points between $\mu_{t r}=[0.0015, \ldots, 0.0035]$ seconds. The variance of the transition density is set to $\sigma_{t r}=2.12 \cdot 10^{-6}$.

The block length for cross-correlation is set to 5000 . The new approach provides results with high accuracy. The estimates of the cross-correlation behave like a stair function. The small steps arise from the discretization of the time delay, because only the maximum value of the crosscorrelation is considered. The height of those steps depend on the sampling frequency.

To reduce the complexity of the new approach the signals can be downsampled first. In the next experiment the two time signals are downsampled with a factor of 10 , thus the sampling frequency is $4800 \mathrm{~Hz}$. If the data is downsampled, the parameter for the transition density has to be adjusted. The transition density can be approximated with a lower number of Gaussians. The transition density is approximated with $N=96$ components. The expected values are linearly equally spaced points between $\mu_{t r}=[0.0015, \ldots, 0.0035]$ seconds and the variance of the transition density is set to $\sigma_{t r}=5.2 \cdot 10^{-6}$.

The block length for cross-correlation is set to 500. The results are shown in Fig. 4. The accuracy is lower for the estimates of the new approach. However, the steps behavior of the estimates of the cross-correlation increases, too, because the sampling frequency is reduced.

\section{CONCLUSIONS AND FUtURE WORK}

A new Bayesian nonlinear filtering technique for estimating unknown parameters from time sequences has been presented and exemplified for time delay estimation. The new approach provides probability density functions describing the parameter estimates that are updated based on every 


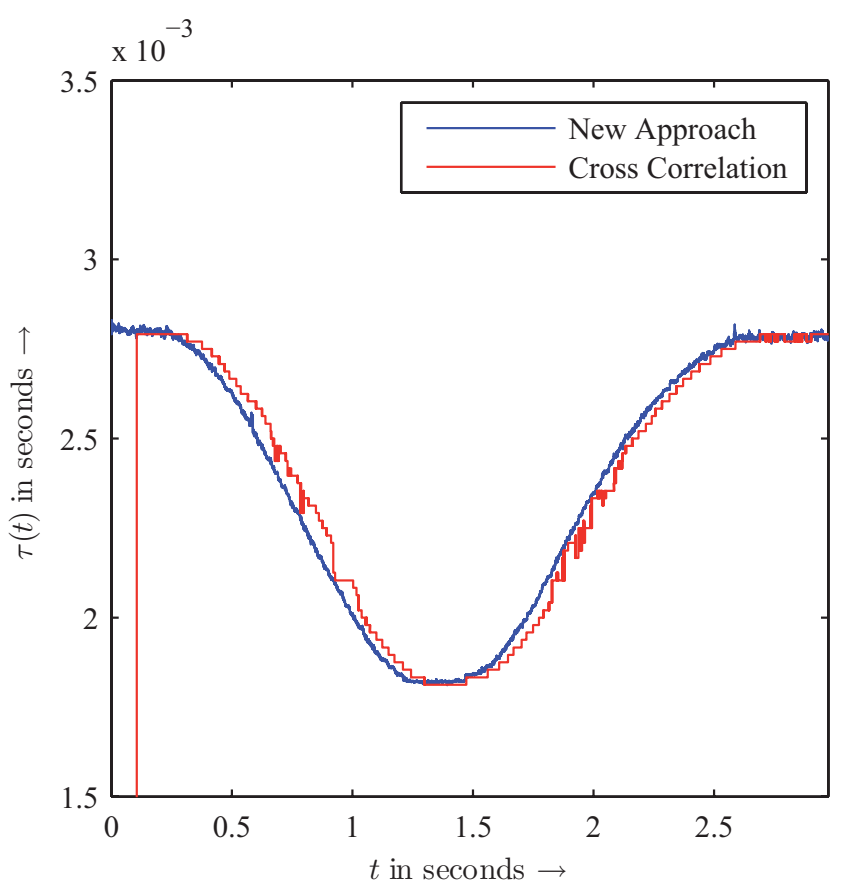

Fig. 3. Experimental result for a moving source. Comparing classic and new approach. The sampling frequency is set to $f_{t}=48000 \mathrm{~Hz}$.

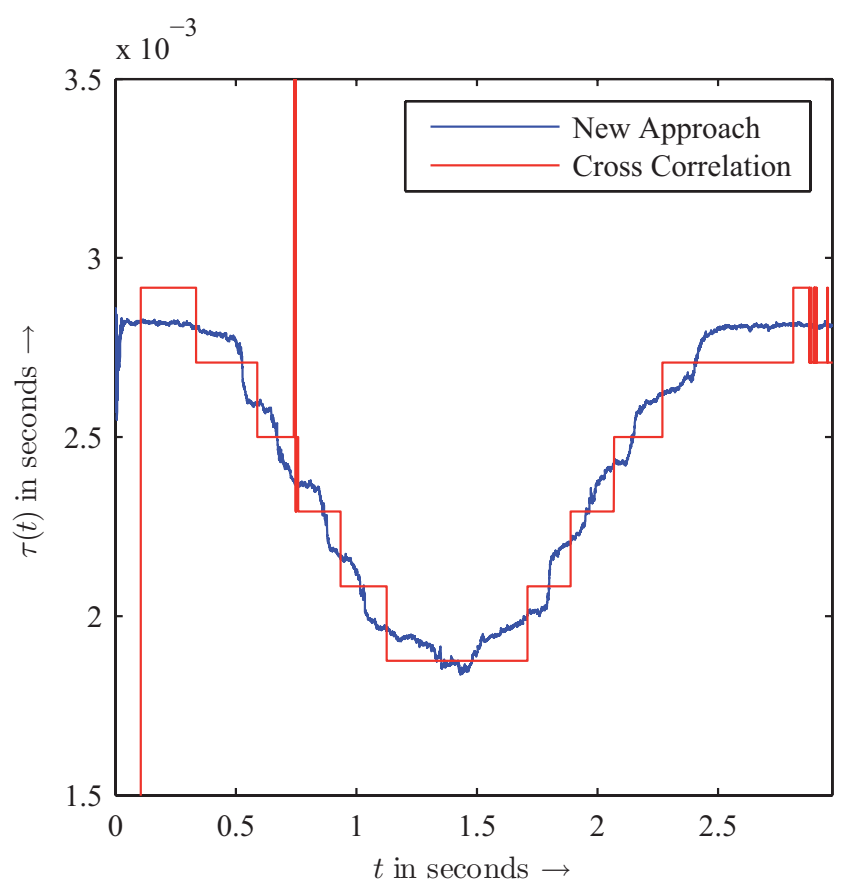

Fig. 4. Experimental result for a moving source. Comparing classic and new approach. The sampling frequency is set to $f_{t}=4800 \mathrm{~Hz}$. received measurement sample. For that purpose, uncertainties in the measurements are explicitly considered. Furthermore, a system model for the time-varying parameters is included.

The complexity of the algorithm is limited, because in the time update the transition density is approximated with an axis-aligned Gaussian mixture. However, the numerical complexity of the new approach is very high, if the sampling frequency increases. Hence, the involved signals are typically downsampled. This results in a decreased accuracy, that is nevertheless still better than the accuracy obtained with the conventional approaches.

Future work is concerned with using the probability density function in a time-of-arrival based localization algorithm, which takes the probabilistic description into account, so that the uncertainties are consequently considered. In addition, a more realistic model as in (4) will be taken into account, where the attenuation term and reflections are considered. If multipath propagation is not considered in the measurement equation, the measurement noise depends on the emitted signal, which has to be considered in future work. Furthermore, a real-time implementation of the algorithm for an acoustic tracking system is currently under development.

\section{REFERENCES}

[1] F. Beutler and U. D. Hanebeck, "Closed-Form Range-Based Posture Estimation Based on Decoupling Translation and Orientation," in Proceedings of IEEE Intl. Conference on Acoustics, Speech, and Signal Processing (ICASSP 05), Pennsylvania, PA, USA, 2005, pp. 989-992.

[2] N. M. Vallidis, "WHISPER: A Spread Spectrum Approach to Occlusion in Acoustic Tracking," Ph.D. dissertation, University of North, Carolina at Chapel Hill, 2002.

[3] C. H. Knapp and G. C. Carter, "The Generalized Correlation Method for Estimation of Time Delay," IEEE Transactions of Acoustics, Speech, and Signal Processing, vol. ASSP-24, no. 4, pp. 320-327, August 1976.

[4] K. Briechle and U. D. Hanebeck, "Template Matching using Fast Normalized Cross Correlation," in Proceedings of SPIE, vol. 4387, 2001.

[5] F. van der Heijden, S. van Koningsveld, and P. Regtien, "Time-ofFlight Estimation using Extended Matched Filtering," in Proceedings of IEEE Sensors 2004, 2004, pp. 1460-1463.

[6] A. Sayed, A. Tarighat, and N. Khajehnouri, "Network-Based Wireless Location: Challenges Faced in Developing Techniques for Accurate Wireless Location Information," in IEEE Signal Processing Magazine, vol. 22, no. 4, 2005, pp. 24-40.

[7] A. Blake, J. Sullivan, M. Isard, and J. MacCormick, "A Bayesian Theory of Multi-Scale Cross-Correlation in Images," in Spatial-temporal modelling and its applications, 18th LASR Workshop, 1999.

[8] J. Sullivan, A. Blake, M. Isard, and J. MacCormick, "Object Localization by Bayesian Correlation," in Proc. Int. Conf. Computer Vision, 1999, pp. 1068-1075.

[9] Y. Lin and D. D. Lee, "Bayesian Regularization and Nonnegative Deconvolution for Time Delay Estimation," in Advances in Neural Information Processing Systems 17, L. K. Saul, Y. Weiss, and L. Bottou, Eds. Cambridge, MA: MIT Press, 2005, pp. 809-816.

[10] M. Huber, D. Brunn, and U. D. Hanebeck, "Closed-Form Prediction of Nonlinear Dynamic Systems by Means of Gaussian Mixture Approximation of the Transition Density," in Proceedings of the IEEE Conference on Multisensor Fusion and Integration for Intelligent Systems (MFI'2006), Heidelberg, Germany, 2006. 